\title{
Clinical Usefulness of Combination Therapy with Minocycline and Ciprofloxacin as a Treatment for Fulminant Cases of Japanese Spotted Fever
}

\author{
Fumihiko Mahara ${ }^{1,2}$, Kazuaki Miyamoto ${ }^{1,2^{*}}$, Hiromi Fujita ${ }^{2}$ and Teruo Mutsuda ${ }^{1}$ \\ ${ }^{1}$ Mahara Hospital, Aratono-cho Nobusato 6-1, Anan, 779-1510, Japan \\ ${ }^{2}$ Mahara Institute of Medical Acarology, Aratano-cho Korekuni 56-3, Anan, 779-1510, Japan
}

*Corresponding author: Kazuaki Miyamoto, Mahara Institute of Medical Acarology, Aratano-cho Korekuni 56-3, Anan, 779-1510, Japan, Fax/Tel: +81-884-36-3601; Email: kazupitts@jtw.zaq.ne.jp

Rec date: Jul 09, 2014; Acc date: Oct 29, 2014; Pub date: Oct 31, 2014

Copyright: ( 2014 Mahara F, et al. This is an open-access article distributed under the terms of the Creative Commons Attribution License, which permits unrestricted use, distribution, and reproduction in any medium, provided the original author and source are credited.

Keywords: Japanese spotted fever; Fulminant cases; Ciprofloxacin; the patient's clinical findings, the suspected clinical diagnosis was Minocycline

\section{Introduction}

Japanese spotted fever (JSF) is a member of the spotted fever group rickettsiosis caused by Rickettsia japonica [1-3]. JSF is transmitted by tick-bite, and clinically characterized with fever, cutaneous eruption (exanthematous legions), and inoculation eschar. Diagnosis of JSF is generally based on clinical presentations, followed by serological confirmation using paired (acute- and convalescent-phase) serum [2]. Recently, only approximately 200 JSF cases have been identified every year in Japan (http://idsc.nih.go.jp/idwr/index.html). However, the endemic regions have been expanded from southwestern area to northern region of Japan (http://idsc.nih.go.jp/idwr/index.html). Occasionally, sufficient therapeutic effects did not been achieved by administration of tetracycline, such as minocycline (MINO) in the treatment of JSF; i.e., fatal JSF cases have been reported even in rapid administration of MINO, while remarkable and rapid improvement of clinical symptoms in cases of scrub typhus is generally obtained by the treatment with MINO. Collectively, empiric but effective antibiotic therapy should be started rapidly and should not await confirmatory testing, even using molecular assays.

In this report, we introduced the more effective treatment than the traditional tetracycline therapy for spotted fever group rickettsiosis in Japan, and also discussed a proposal mechanism of the combination therapy; anti-rickettsial effects and an additional effect(s) against hypercytokinemia which is based on molecular action by minocycline [4].

\section{Case 1}

On 23 April, a 78-year-old woman was admitted to Mahara hospital with a fever and disturbances of consciousness. On 22 April, she had experienced a mild fever $\left(\sim 37.5^{\circ} \mathrm{C}\right)$ and general fatigue. By the day of admission, her body temperature had risen to $39.4^{\circ} \mathrm{C}$ and her systolic blood pressure was $68 \mathrm{mmHg}$, which was considered to represent a pre-shock state. The patient's consciousness level was defined as stupor. Many exanthematous skin legions of 1 to $8 \mathrm{~mm}$ in diameter were observed on the patient's trunk and extremities, including her palms and soles, and a tick bite eschar was observed in her left lumbar region. The patient's C-reactive protein level was markedly increased $(17.5 \mathrm{mg} / \mathrm{dL})$. However, a blood cell count did not detect anemia, leukocytosis $(6330 / \mu \mathrm{L})$, but detected thrombocytopenia $(7.4 \times 104 / \mu \mathrm{L})$. In addition, biochemical examinations demonstrated that the patient's aspartate transaminase (AST) and alanine transaminase (ALT) levels were slightly increased to $42 \mathrm{U} / \mathrm{L}$ and $48 \mathrm{U} / \mathrm{L}$, respectively. Based on Japanese spotted fever (JSF) complicated with disseminated intravascular coagulation (DIC) and encephalopathy. Minocycline (MINO) (200 mg/day) was administered intravenously, and methylprednisolone $(1,000 \mathrm{mg} /$ day $)$ and dopamine were also administered as treatments against JSF-associated hypercytokinemia [5]. During following four days, the patient's body temperature fell below $37^{\circ} \mathrm{C}$. However, on the fourth day after admission her body temperature had increased to $38^{\circ} \mathrm{C}$, and after start of MINO administration, severe general fatigue and appetite loss were continued. Therefore, ciprofloxacin (CPFX) (600 mg/day) was added to MINO therapy. The patient was afebrile on the 9th day after admission. On 30 April, an immunoperoxidase (IP) assay indicated that the patient's titers of IgG and IgM antibodies against $R$. japonica were 40 and 1280 , respectively.

\section{Case 2}

On 14 June, a 66-year-old woman suffered from a fever of more than $38^{\circ} \mathrm{C}$ and general fatigue. On 17 June, the fever remained, and skin eruptions ( $\sim 5 \mathrm{~mm}$ in diameter) appeared on her trunk. She was admitted to hospital on 19 June, at which point her body temperature was $\sim 41^{\circ} \mathrm{C}$. Antibiotic therapy with piperacillin sodium ( $4 \mathrm{~g} /$ day), was started under a diagnosis of fever of unknown origin. On 20 June, she was diagnosed with a suspected rickettsial infection based on her fever and skin eruptions, and MINO (200 mg/day) treatment was started. On 21 June, she was transferred to our hospital with a fever, mild respiratory failure, and generalized edema. At this point, her body temperature was $39.8^{\circ} \mathrm{C}$, and exanthematous legions that were typical of those seen in JSF were observed. Several tick bite eschars were also found on her legs. In laboratory examinations, a normal white blood cell count $(7190 / \mu \mathrm{L})$ and thrombocytopenia $(6.1 \times 104 / \mu \mathrm{L})$ were detected. In addition, the patient's C-reactive protein level was markedly increased to $24.8 \mathrm{mg} / \mathrm{dL}$, and her fibrinogen degradation product (FDP) concentration was $76.0 \mathrm{mg} / \mathrm{dl}$. Furthermore, she displayed elevated transaminase levels (AST: $67 \mathrm{U} / \mathrm{L}$; ALT: $52 \mathrm{U} / \mathrm{L}$ ). These findings indicated that the patient suffered from fulminant JSF complicated with DIC. And also, chest $\mathrm{x}$-ray showed pneumonia pattern. Thus, MINO (200 mg/day, intravenously) and CPFX (300 mg/ day, orally) were administered simultaneously. During the first six days after admission, the patient's body temperature remained at 38 to $39^{\circ} \mathrm{C}$. However, her body temperature gradually fell from the 7 th day after admission, and she became afebrile by the 10th day after admission. In serological examinations based on IP assays, tests for IgG and IgM antibodies against $R$. japonica were negative (less than 8 ) on the day of admission, but titers rose to 640 and 1,280, respectively, on the 9th day after admission. 
Japanese spotted fever (JSF), which is a type of spotted fever group rickettsiosis seen in Japan, was first recognized by F. Mahara in 1984 [6,7]. The causative pathogen was subsequently named Rickettsia japonica [3]. The most important vectors for transmitting the pathogen are Haemaphysalis longicornis, H. flava, and H. hystericus $[1,2,8]$. Despite its name, this arthropod-borne disease is rare in Japan; i.e., less than 200 cases occurred each year from 1999 to 2011 (http:// idsc.nih.go.jp/idwr/index.html). Regarding the clinical features of the JSF, patients sometimes only complain of fever and mild malaise during the first 2-3 days, but subsequently suffered from a spiking fever (usually more than $39^{\circ} \mathrm{C}$ ) combined with severe malaise $[1,2]$. Therefore, determining the most effective antibiotic treatment based on an accurate clinical diagnosis is difficult without finding tick bite lesions, which are present in most cases and are usually smaller than those seen in scrub typhus (in contrast to those found in cases of Rocky Mountain spotted fever) [1,2]. In addition, typical exanthematous legions develop on the extremities and face, especially the palms and soles, and spread to the trunk. The appearance of such legions is useful for diagnosis, and they usually develop 2 to 10 days after a tick bite or 2 to 3 days after the abrupt onset of fever $[1,2]$. While a triad of fever, skin eruptions, and eschars is useful for diagnosing JSF, these symptoms are seldom found in the early phase of the disease. Thus, serological and/or PCR assays using biopsy specimen(s) of tick-bite eschar(s) or skin biopsy specimens are often necessary to confirm a diagnosis of JSF; however, such diagnostic assays are not available in clinical practice in Japan [1,2]. In severe cases, one or more of DIC, central nervous system infection (meningoencephalitis), acute respiratory distress syndrome, septic shock and/or multi-organ failure (MOF) is present even in the early phase of the disease, with or without the typical symptoms of JSF [5,9]. Among fulminant cases of JSF, some fatalities have been reported, which were mainly caused by the delay of appropriate antibiotic treatment [10, personal communication]. To avoid death or serious complications due to JSF, the administration of antibiotics must be started as soon as possible in cases of JSF which is suspected based on clinical findings. However, diagnosis based on clinical symptoms alone in the acute phase of the condition (fever and mild malaise) and differentiating JSF from other febrile diseases caused by viruses can be difficult, even in endemic areas, because of the rarity of JSF. Concerning antibiotic treatment, tetracyclines, especially MINO, are the most effective antibiotics, whereas penicillin and cephalosporin antibiotics are not effective against JSF rickettsia, which is also true for other rickettsioses $[1,2]$.

In case 1, CPFX was administered in addition to MINO because the patient's fever recurred and only a limited improvement in the patient's general condition had been observed during the first 7 days of MINO treatment. The patient was afebrile within two days of the start of CPFX treatment. Case 2 was a case of fulminant JSF complicated with MOF and DIC. Combination therapy involving MINO and CPFX might have been effective because the patient's body temperature subsided during the 7-day treatment period. Moreover, we know of another three cases of JSF to whom the new quinolone (NQ) antibiotic tosufloxacin was administered. Of these three cases, one case was cured using tosufloxacin treatment alone. However, a fatal case of JSF complicated with DIC and MOF which condition was treated with a
NQ antibiotic has also been reported. In the latter case, MINO and the NQ antibiotic were administered from a few days after the onset of the illness; i.e., the combination therapy was delayed [9]. These cases indicate that CPFX might be an effective antibiotic for use in combination with MINO. In the literature, two $R$. japonica isolates from patients that were diagnosed at our hospital were found to be sensitive to CPFX and other NQ antibiotics in vitro [11]. A recent report indicated that $\mathrm{MINO}$ can induce cytokine modulation; i.e., it stated that the simultaneous administration of MINO and CPFX not only represents the use of a combination of bacteriostatic and bacteriocidal drugs, but also indicates a combination of antimicrobial and cytokine-modulating drugs [4]. In addition, a previous clinical study suggested that combination therapy involving MINO and one of the NQ antibiotics might be effective in pediatric cases of JSF [12].

Overall, MINO combined with NQ antibiotic therapy should be rapidly administered as a treatment for cases of JSF involving signs of severe complications. Due to their antibacterial activity and cytokinemodulating effects, combination therapy with both of these antibiotics is a useful strategy for early stage febrile tick-borne diseases.

\section{References}

1. Mahara F (1997) Japanese spotted fever: report of 31 cases and review of the literature. Emerg Infect Dis 3: 105-111.

2. Mahara F (2006) Rickettsioses in Japan and the far East. Ann N Y Acad Sci 1078: 60-73.

3. Uchida T, Uchiyama T, Kumano K, Walker DH (1992) Rickettsia japonica sp. nov., the etiological agent of spotted fever group rickettsiosis in Japan. Int J Syst Bacteriol 42: 303-305.

4. Tai K, Iwasaki H, Ikegaya S, Ueda T (2013) Minocycline modulates cytokine and chemokine production in lipopolysaccharide-stimulated THP-1 monocytic cells by inhibiting IкB kinase $\alpha / \beta$ phosphorylation. Transl Res 161: 99-109.

5. Iwasaki H, Mahara F, Takada N, Fujita H, Ueda T (2001) Fulminant Japanese spotted fever associated with hypercytokinemia. J Clin Microbiol 39: 2341-2343.

6. Mahara F (1984) Three Weil-Felix reaction (OX2) positive cases with skin eruption and high fever. J Anan Med Assoc 68: 4-7.

7. Mahara F, Koga K, Sawada S, Taniguchi T, Shigemi F, et al. (1985) The first report of the rickettsial infections of spotted fever group in Japan: three clinical cases. Kansenshogaku Zasshi 59: 1165-1171.

8. Mahara F (2008) Japanese spotted fever: History of discovery, clinical investigations and current epidemiology. Modern Media 54: 32-41.

9. Kodama K, Senba T, Yamauchi H, Nomura T, Chikahira Y (2003) Clinical study of Japanese spotted fever and its aggravating factors. J Infect Chemother 9: 83-87.

10. Wada K, Sakaeda H, Aono R, Chiya S (2008) [Fulminant Japanese spotted fever--the second fatal case in Japan]. Kansenshogaku Zasshi 82: 77-81.

11. Miyamura S and Ohta T (1991) In vitro susceptibility of rickettsial strains from patients with Japanese Spotted Fever to quinolones, penicillins and other selected chemotherapeutic agents. Jpn J Chemother (CHEMOTHERPY) 39: 258-260.

12. Nashida Y, Higashigawa M, Maegawa K, Fujiwara T, Inoue M (2011) Clinical investigation of nine pediatric Japanese spotted fever cases. Kansenshogaku Zasshi 85: 638-643. 